\title{
Retrograde Approach through the Posterior Communicating Artery and Anterior Communicating Artery to the Ophthalmic Artery
}

W e read the article about alternative routes in intraocular chemotherapy for retinoblastoma by Bertelli et al, ${ }^{1}$ published in July 2016, with considerable interest. It gives excellent knowledge about intra-arterial chemotherapy of the retinoblastoma. The authors have mainly described 3 patterns of drug delivery: a fixed pattern through the ophthalmic artery, a fixed pattern through branches of the external carotid artery, and a variable pattern via either of these. They have also emphasized difficulties in direct ophthalmic artery catheterization because of its acute takeoff from the internal carotid artery.

We additionally want to mention an alternative route for the superselective catheterization of the ophthalmic artery, which was published in 2014 . $^{2}$ We performed 29 intra-arterial chemotherapy sessions for 12 unilateral retinoblastomas. In the first 4 patients and 10 sessions, we used the ipsilateral ICA to reach the ophthalmic artery of the affected globe (classic ipsilateral approach). Then, we realized that the retrograde approach by the posterior communicating artery that was located just opposite the wall of the ICA or anterior communicating artery seemed easier and reasonable. We have also known that the anterior communicating artery and both of the posterior communicating arteries are patent in neonates and children. So, we used the vertebral artery, basilar artery, ipsilateral/contralateral P1 segment of the posterior cerebral artery, and ipsilateral/contralateral posterior communicating artery, respectively, to reach inside the ophthalmic artery at the next 15 sessions (retrograde approach). At 4 sessions, we used the anterior communicating artery to reach the contralateral ophthalmic artery. The angle between the ophthalmic segment of the ICA and the proximal segment of the ophthalmic artery became more convenient for catheterization (average angle at ipsilateral

http://dx.doi.org/10.3174/ajnr.A5081 approach, $53.4^{\circ}$; average angle at retrograde approach, $\left.148.3^{\circ}\right)$. In addition, the fluoroscopy total time was diminished compared with the classic approach (mean time at ipsilateral approach, 21.5 minutes; mean time at retrograde approach, 7.5 minutes). We did not have any high-degree stenotic or occluded ophthalmic arteries because of previous treatments. It may be because of the smaller number of our sessions (maximum sessions for an ophthalmic artery was 3 ). In such cases, the alternative routes and strategies that have been published by Bertelli et $\mathrm{al}^{1}$ are very important.

If the ophthalmic artery is patent, the retrograde approach makes the intra-arterial chemotherapy procedure easier in patients with retinoblastoma and shortens the fluoroscopy total time.

\section{REFERENCES}

1. Bertelli E, Leonini S, Galimberti D, et al. Hemodynamic and anatomic variations require an adaptable approach during intra-arterial chemotherapy for intraocular retinoblastoma: alternative routes, strategies, and follow-up. AJNR Am J Neuroradiol 2016;37:1289-95 CrossRef Medline

2. Saglam M, Sarici A, Anagnostakou V, et al. An alternative technique of the superselective catheterization of the ophthalmic artery for intraarterial chemotherapy of the retinoblastoma: retrograde approach through the posterior communicating artery to the ophthalmic artery. Neuroradiology 2014;56:751-54 CrossRef Medline

(D) M. Saglam Department of Radiology Dr. Siyami Ersek Chest and Cardiovascular Surgery Hospital Istanbul, Turkey (1) O. Kizilkilic Department of Radiology Division of Neuroradiology Istanbul University Cerrahpasa Faculty of Medicine Istanbul, Turkey 